\title{
Probe of Dark Galaxies via Disturbed/Lopsided Isolated Galaxies
}

\author{
Igor D. Karachentsev ${ }^{1}$, Valentina E. Karachentseva ${ }^{2}$, Walter K. \\ Huchtmeier $^{3}$, Dmitry I. Makarov ${ }^{1}$, and Serafim S. Kaisin ${ }^{1}$ \\ ${ }^{1}$ Special Astrophysical Observatory, Russian Academy of Sciences, N.Arkhyz, KChR, \\ 369167, Russia \\ email:ikar@sao.ru,dim@sao.ru,skai@sao.ru \\ ${ }^{2}$ Astronomical Observatory of Kiev University, Observatorna 3, $04053 \mathrm{Kiev}$, Ukraine, \\ email:vkarach@observ.univ.kiev.ua \\ ${ }^{3}$ Max-Planck-Institut für Radioastronomy, Auf dem Hügel 69, D-53121 Bonn, Germany \\ email:p083huc@mpifr-bonn.mpg.de
}

\begin{abstract}
Searching for lopsided/interacting objects among 1500 isolated galaxies yields only eight strongly disturbed galaxies which may be explained as a result of their interaction with massive dark objects. We present results of spectral and photometric observations of these galaxies performed with the 6-m telescope that lead to significant restriction on cosmic abundance of dark galaxies.
\end{abstract}

Keywords. dark matter, interaction galaxies, peculiar galaxies

\section{Introduction}

The standard Lambda-CDM cosmology assumes that besides dark halos with luminous galaxies in their cores, completely dark clumps (sub-halos) should also exist with masses $10^{8}-10^{11} M_{\odot}$ (van den Bosch et al. 2003, Tully 2005, Yang et al. 2005). The total number of dark sub-halos may exceed the number of the usual galaxies by a factor of tens. Within the Local Group, Klypin et al. (1999) predict about 300 satellites with masses greater than $3 * 10^{8} M_{\odot}$ that is significantly higher than the observed one.

A possible explanation for this discrepancy is the physical processes inhibiting star formation especially in low mass clumps, thus implying the existence of a large number of dark satellites.

Here, we use an approach proposed by van den Bergh (1969) and Trentham et al. (2001), which relies on searching for signs of non-motivated distortion visible on images of spatially isolated galaxies.

We know galaxies in close encounters show a significant signature of interaction in the form of a distortion of their structure, the presence of tails and bridges, or a common diffuse envelope. All these features have been quantitatively explained based on numerous N-body simulations ever since Toomre \& Toomre (1972).

Signs of interaction are seen in more than $50 \%$ of those binary galaxies where the separation is comparable to the sum of the diameters. In systems with greater separation, as in triplets, the relative number of peculiar galaxies is about 30\%. In magnitude-limited galaxy catalogs, the fraction of interacting objects is equal to $8 \%$ (Karachentsev, 1987).

When considering more and more scattered systems of galaxies and single galaxies of the "general field", one can expect a fraction of the interacting objects among them to 
be nearby zero. This would occur if there were no other objects except the luminous galaxies.

However, if completely dark galaxies with masses of $10^{8}-10^{11} M_{\odot}$ exist, the phenomena of interaction will occur in the case of extremely isolated galaxies too. Hence, an asymptotic relative number of peculiar shapes among the most spatially isolated galaxies may be a sensitive tool for estimating the cosmic abundance of massive dark galaxies.

\section{Searching for peculiar isolated galaxies}

To search for such strange cases of interaction where the second interacting companion is invisible, we used the "Catalog of Isolated Galaxies" (Karachentseva $1973=$ KIG). This catalog contains 1050 galaxies without "significant" neighbors.

According to our estimates, the catalog objects do not suffer essential perturbations from neighboring galaxies over some Gyrs. As it is a sample of northern galaxies with $m<15.7$, the catalog includes only $4 \%$ of the CGCG galaxies from Zwicky et al. (19611968); hence KIG is a collection of a rather rare kind of galaxies.

All KIG galaxies were inspected by us on the Digital Sky Survey. In some cases, we also studied galaxy images in the 2MASS to check for the possibility of double-nuclei systems as recent merging remnants. The results of our inspections (Karachentsev et al. 2006) yield five disturbed/lopsided galaxies.

Then, we undertook a new search for isolated distorted galaxies in a sample limited by radial velocities $V_{L G}<3200 \mathrm{~km} \mathrm{~s}^{-1}$. Among 7500 such galaxies of the Local Supercluster, about $60 \%$ reside in groups of different populations (Makarov \& Karachentsev, 2000). The remaining $N \sim 3000$ galaxies are characterized by different degrees of isolation with respect to their neighbors.

We selected 500 of the most isolated galaxies and inspected their images on DSS. Only 4 galaxies out of 500 show significant signs of interaction. One case, UGC 4722, turns out to be common with the KIG sample.

Signs of interaction between galaxies are best developed when the objects have similar masses. If the number of completely dark sub-halos with typical masses of $10^{8}-10^{11} M_{\odot}$ in any volume approximately corresponds to the number of luminous galaxies, then one may expect about $8 \%$ interacting galaxies among the isolated ones (interaction with an invisible object). This rough estimate ignores, of course, properties of spatial distribution of dark sub-haloes with respect to the luminous galaxies.

The observed relative number of KIG galaxies with clear features of interaction, $5 / 1050=0.5 \%$, turns out to be one order of magnitude lower than expected.

The observed frequency of disturbed shapes among very isolated galaxies in the Local Supercluster, $4 / 500=0.8 \%$, is consistent with the previous estimate made for the KIG sample. In view of these results, it is rather unlikely that the number of massive dark sub-halos is similar to the number of the usual galaxies.

Moreover, apart from interaction with a dark galaxy, the observed morphological irregularities of isolated galaxies may have different origins, in particular, there could have been a merger, with the companion now merged and not visible anymore, or there could be large gas accretion from cosmic filaments, leading to perturbed morphologies via star bursts.

As noted by Trentham, dark galaxies probably have low masses and negligible dynamical effects on massive galaxies like the ones in the KIG sample. They can have much more substantial effects on the nearby low-mass galaxies seen, say, in the Catalog of Neighboring Galaxies (Karachentsev et al. 2004). 
Table 1. Isolated galaxies with disturbed structure

\begin{tabular}{lcccc}
\hline Galaxy & $V_{L G}$ & $M_{B}$ & $\log \left(L_{B}\right)$ & $\log (\mathrm{MHI})$ \\
\hline \multicolumn{5}{c}{ Probable mergers: } \\
UGC 5101 & +11904 & -21.1 & 10.60 & - \\
UGC 11905 & +7737 & -20.6 & 10.40 & - \\
UGC 11871 & +8230 & -21.0 & 10.56 & 9.68 \\
F635-2 & $+12000:$ & -20.7 & 10.44 & - \\
Asymmetric SF: \\
ESO 539-7 & +3279 & -18.6 & 9.60 & 9.74 \\
NGC 244 & +1021 & -17.0 & 8.96 & 8.73 \\
UGC 4722 & Probably disturbed by a dark galaxy: & \\
ESO 545-5 & +1705 & -16.9 & 8.92 & 9.41 \\
\hline \multicolumn{7}{c}{+2329} & -18.9 & 9.72 & 9.89 \\
\hline
\end{tabular}

This sample contains 197 quite isolated galaxies with a "tidal index" $T I<0$. (A negative $T I$ means that the Keplerian cyclic period of the galaxy with respect to its main neighboring disturber exceeds the cosmic Hubble time.) About $90 \%$ of them are low mass dwarfs.

Pustilnik et al. (2005) found that an isolated nearby galaxy, DDO 68 with $M_{B}=-14.3$ and $T I=-1.6$, looks like a disturbed object with a long curved tail. If DDO 68 is a single such object in the Local Volume, it yields a fraction of disturbed isolated galaxies, $1 / 197=0.5 \%$, the same as for the KIG catalog.

\section{Observations with the BTA 6-m telescope}

Spectral observations were performed by us for some faint galaxies around each of the eight peculiar objects. No physical companions to the considered peculiar galaxies were found, confirming their high isolation.

The peculiar galaxies were imaged in $B, V, R$ bands, and in the emission $H_{\alpha}$ line. Longslit spectra were obtained also across the target galaxies to study their kinematics. For the observations we used the multi-mode focal reducer SCORPIO installed at the BTA $6-\mathrm{m}$ telescope. Figure 1 presents images of 8 the galaxies in different optical bands. The right hand images are given in larger scale for better seeing of the central regions.

Preliminary analysis of the data allows us to separate eight the galaxies into three categories, as shown in Table 1 :

A) Galaxies of high luminosity with ripples, plumes and loops, caused apparently by a recent merging; two of them, UGC 5101 and UGC 11905, have been classified as mergers by Rothberg \& Joseph (2004) based on K-band surface photometry.

B) Galaxies of low luminosity with asymmetric star formation (ESO 539-7 and NGC 244); they have a quite regular undisturbed periphery and eccentric HII regions.

C) Galaxies (UGC 4722 and ESO 545-5), whose disturbed peculiar shape may be really originated by a tight encounter with a massive dark object. Note that both the galaxies have hydrogen mass-to-luminosity ratio, $M(H I) / L_{B}$, higher than 1 in solar units. Results of our spectral and photometric observations for all eight the galaxies will be discussed in details in a separate paper.

Finally, we suggest that the existence of dark galaxies can be a probable reason of signs of interactions observed in some isolated galaxies. However, a cosmic abundance of dark galaxies is likely less than $1 / 20$ the population of usual luminous galaxies. 

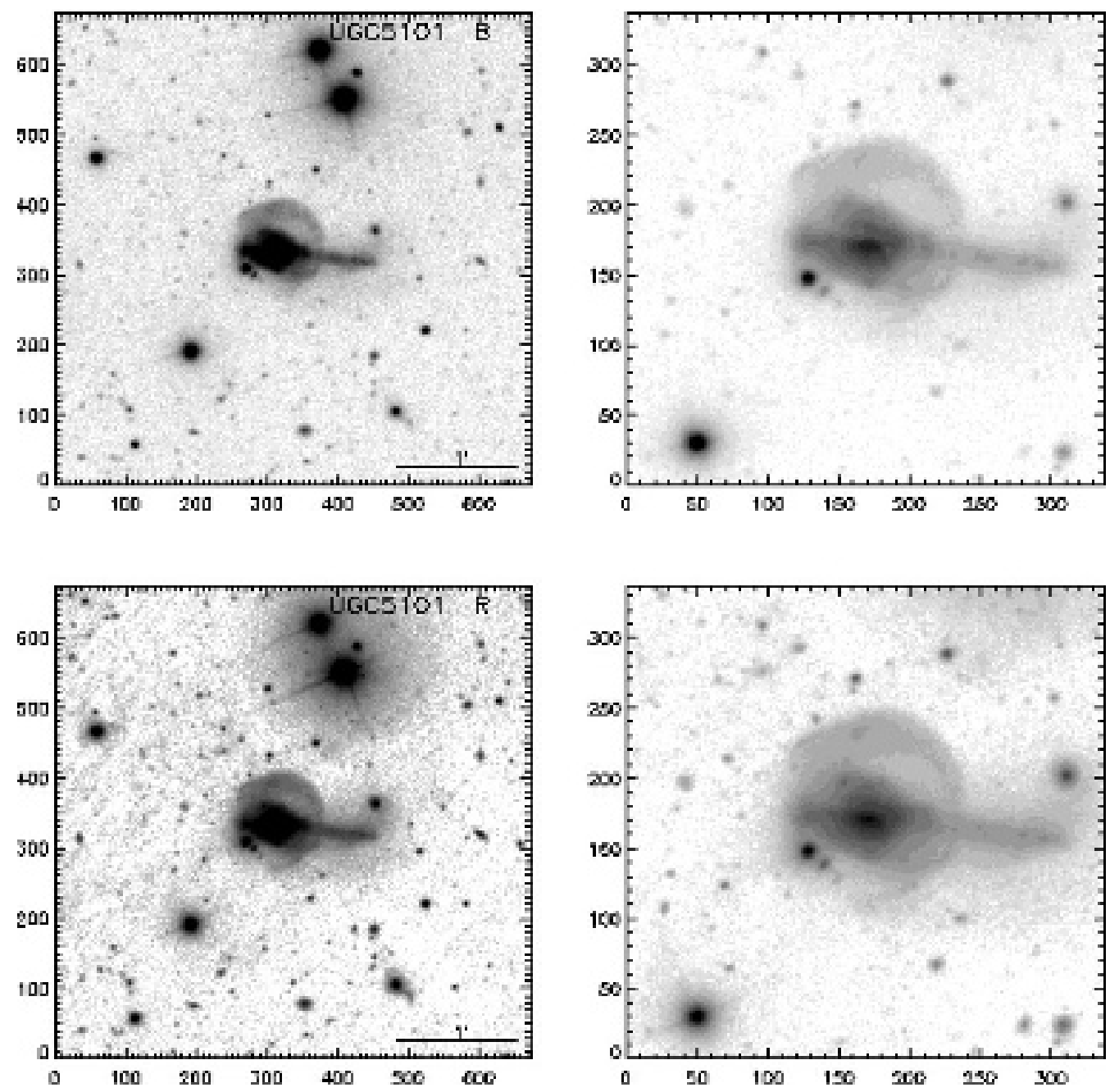

Figure 1. BTA images of the eight disturbed isolated galaxies seen in different optical bands. Enlarged central parts of them are given at right side. East is left and North is up. 

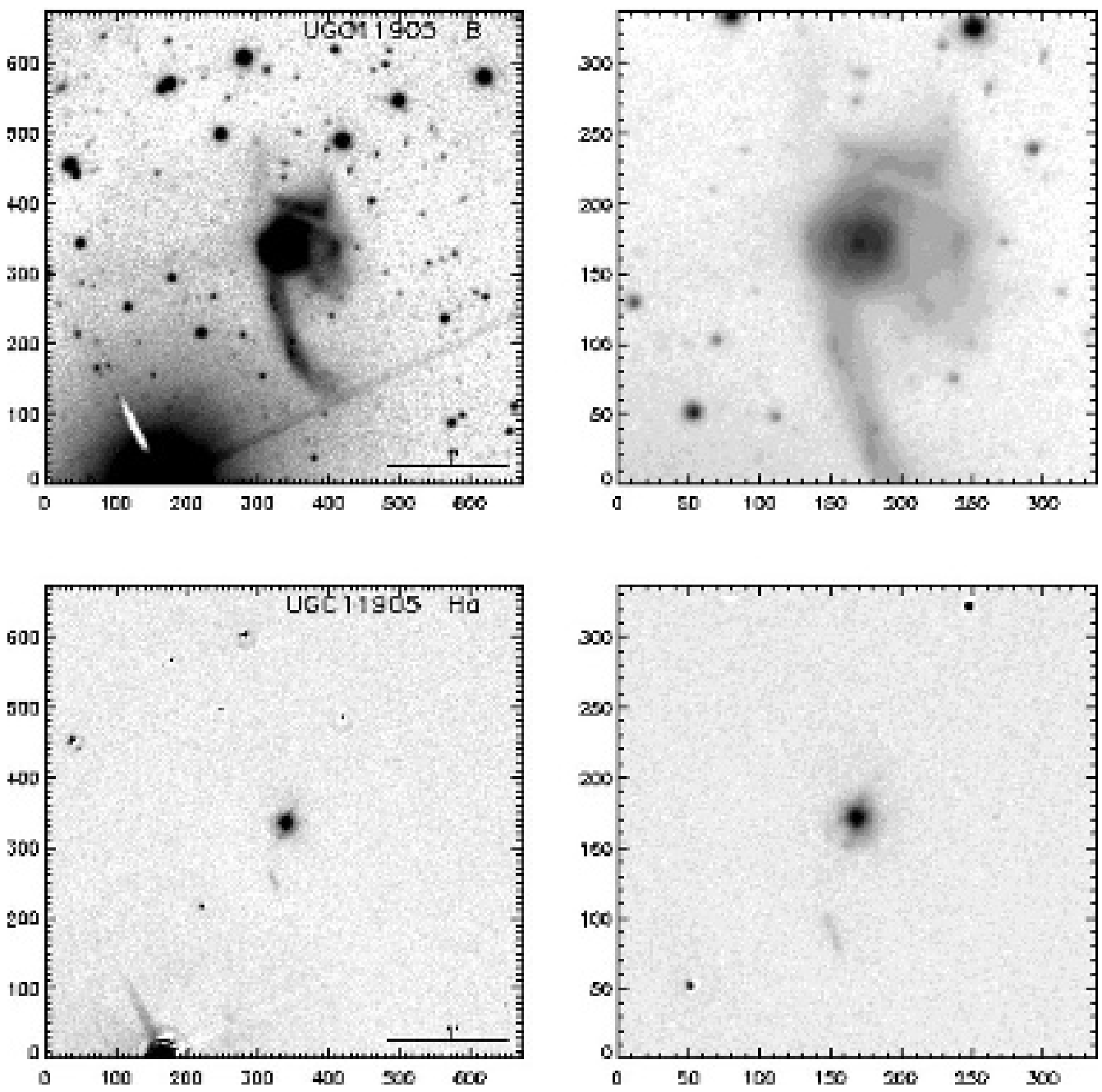

Figure 1. Continued 

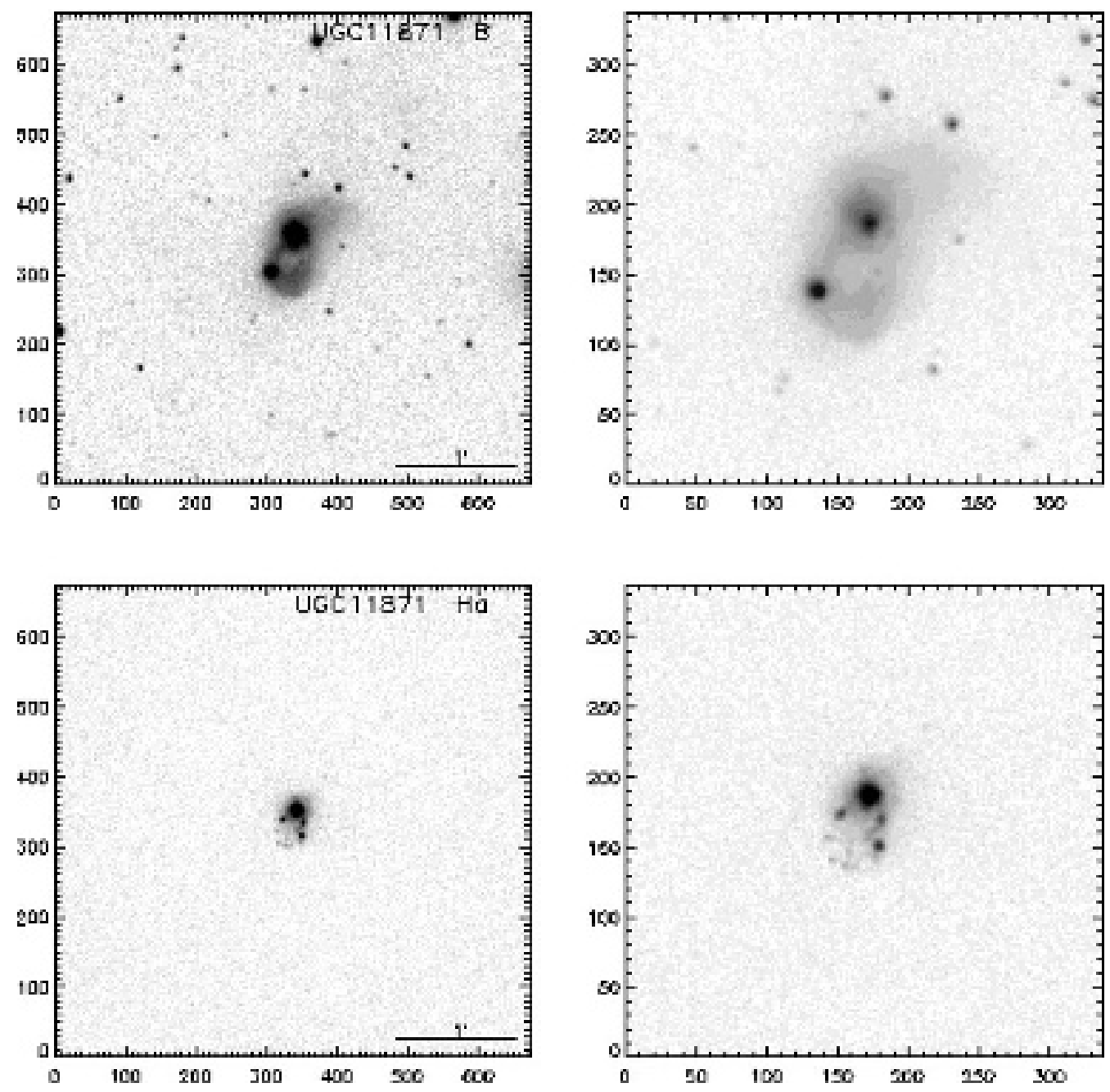

Figure 1. Continued 

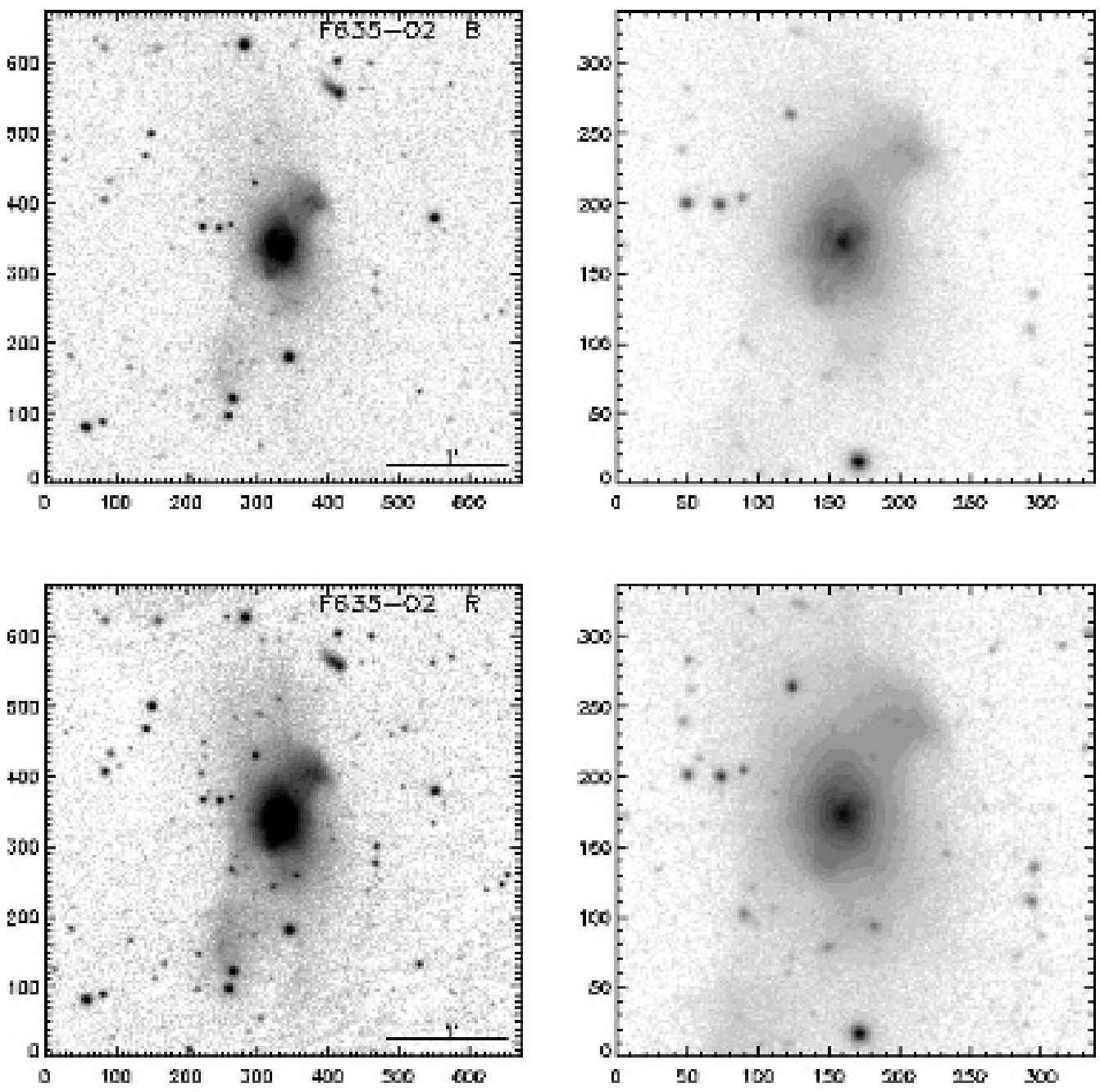

Figure 1. Continued 

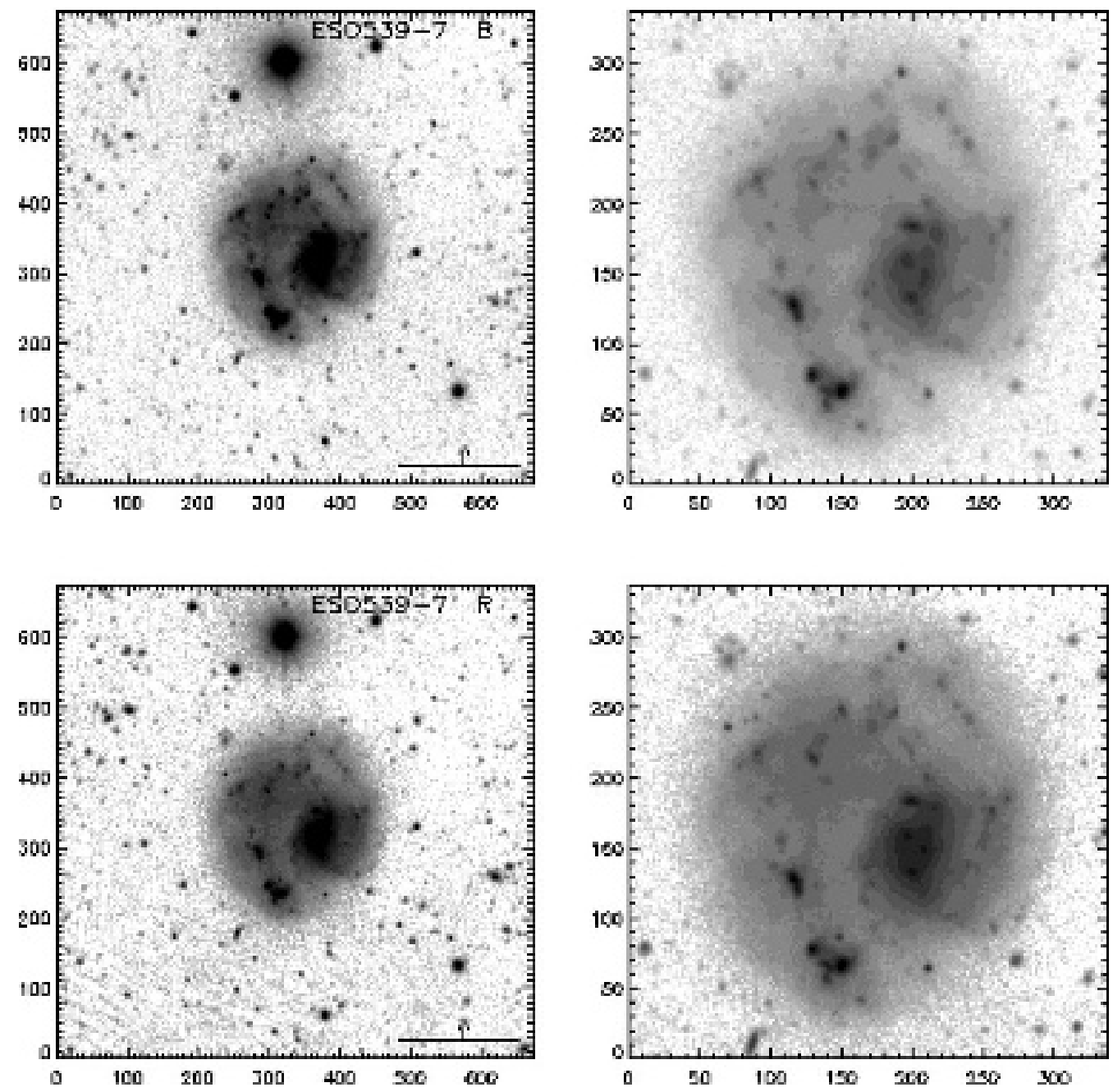

Figure 1. Continued 

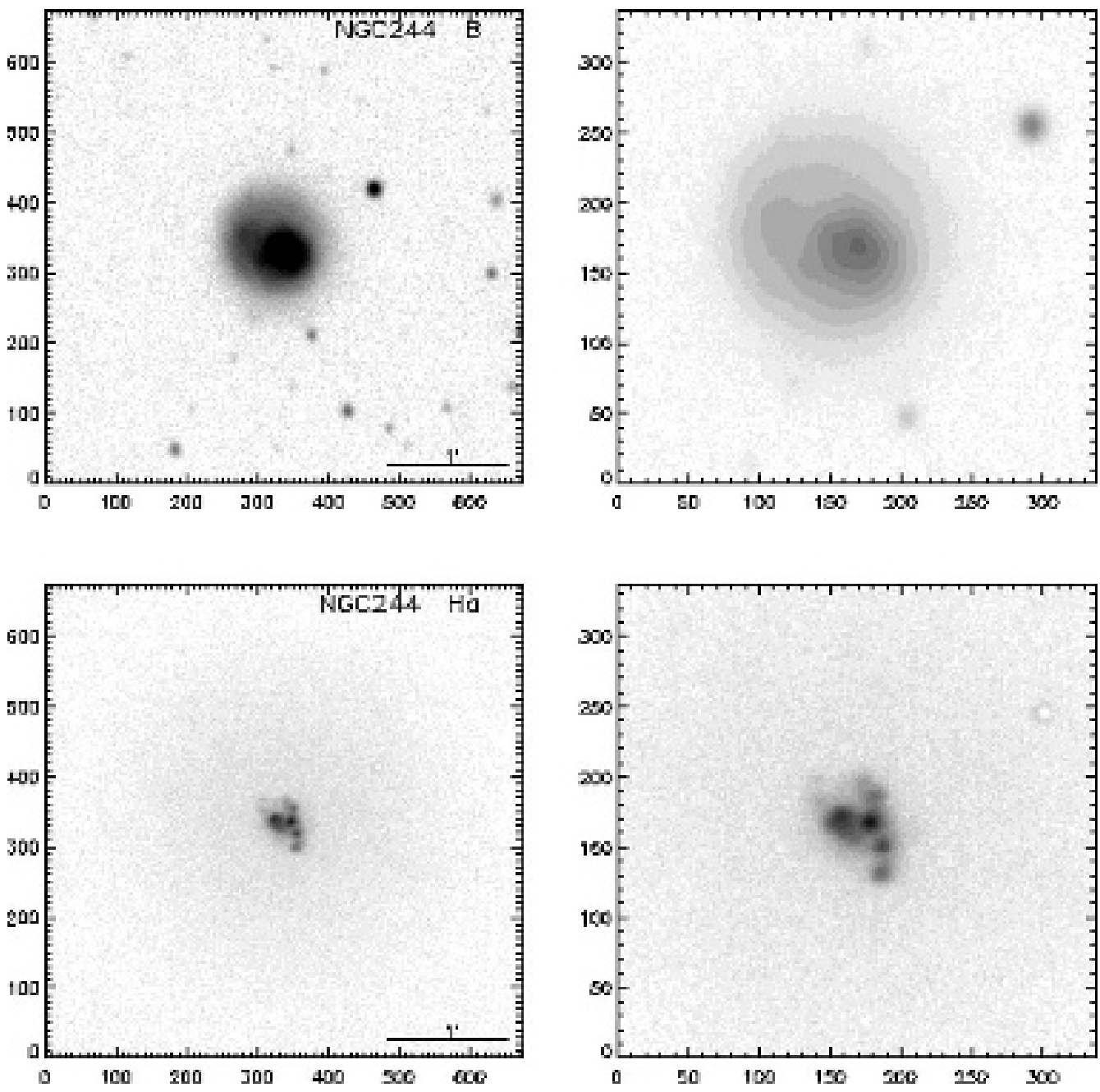

Figure 1. Continued 
I. Karachentsev et al.
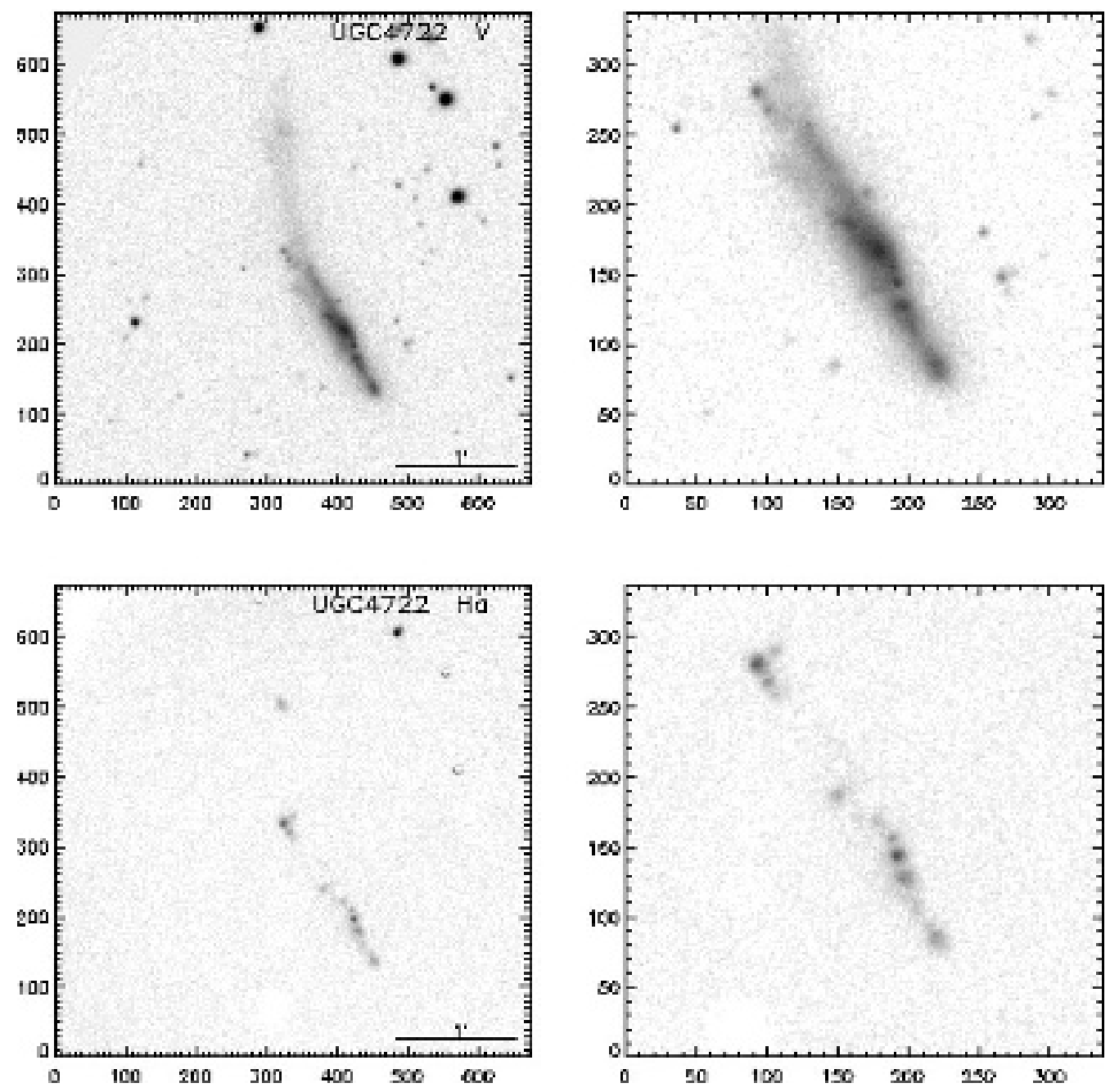

Figure 1. Continued 

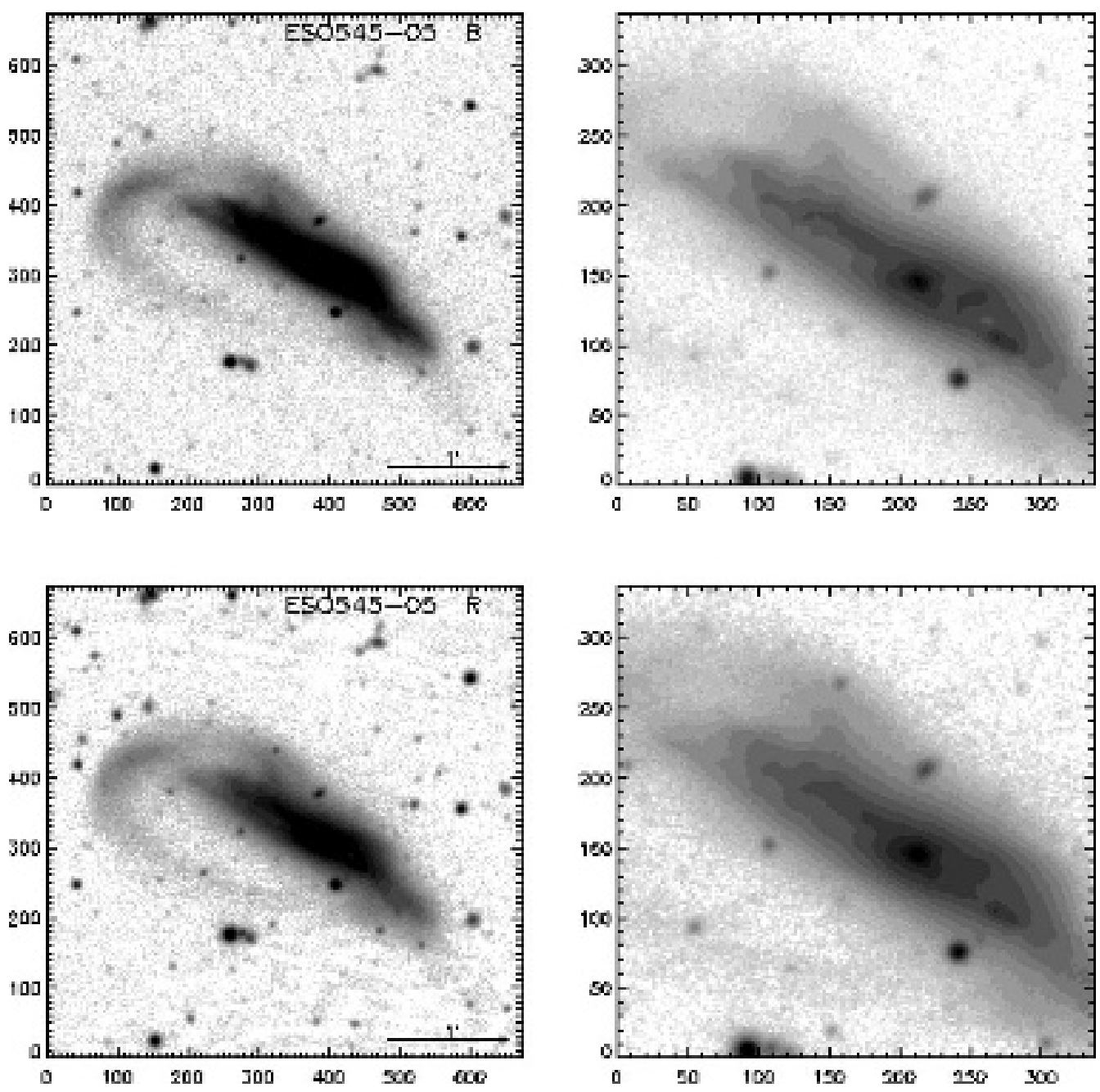

Figure 1. Continued 
Acknowledgements This work was supported by DFG-RFBR grant 06-02-04017

\section{References}

Karachentseva, V. E. 1973, Soobsch. of Spec. Astrop. Obs. 8, 3

Karachentsev, I. D. 1987, Binary Galaxies, Moscow, Nauka

Karachentsev, I. D., Karachentseva, V. E., Huchtmeier, W. K. \& Makarov, D. I. 2004, AJ 127, 2031

Karachentsev, I. D., Karachentseva, V. E. \& Huchtmeier, W. K. 2006, A\&A 451, 817

Klypin, A. A., Kravtsov, A. V. \& Valenzuela, O. 1999, ApJ 522, 82

Makarov, D. I. \& Karachentsev, I. D. in "Small Galaxy Groups", ASP Conf. Series, 2000, v. 209, p.40, eds. M.Valtonen, C.Flinn

Pustilnik, S. A., Kniazev, A. Y. \& Pramskij, A. G. 2005, A\&A 443, 91

Rothberg, B. \& Joseph, R. D. 2004, AJ 128, 2098

Toomre, A. \& Toomre, J. 1972, ApJ 178, 623

Trentham, N., Moller, O. \& Ramires-Ruiz, E. 2001, MNRAS 322, 658

Tully, R. B. 2005, ApJ 618, 214

van den Berg, S. 1969, Nature 224, 891

van den Bosch, F., Yang, X. \& Mo, H. J. 2003, MNRAS 340, 771

Zwicky, F. et al. 1961-1968, Catalog of Galaxies and of Clusters of Galaxies, California, Pasadena, (CGCG)

Yang, X., Mo, H. J., Jing, Y. P. \& van den Bosch, F. 2005, MNRAS 358, 217 